\title{
Risk assessment and prognostic aspect of coagulopathy in Coronavirus Disease 2019 (COVID-19)
}

\author{
MUJIBUR RAHMAN ${ }^{1}$ and Nadira Naznin Rakhi ${ }^{2}$ \\ ${ }^{1}$ BSMRSTU \\ ${ }^{2}$ Bangabandhu Sheikh Mujibur Rahman Science and Technology University
}

May 22, 2020

\begin{abstract}
Since December 2019, the rapid spread of SARS CoV-2 across the border, shuffled into a world pandemic situation with an alarming rate of morbidity and mortality. Concerns are mounting as the reports indicate tangled circumstances among the COVID-19 patients due to blood coagulopathy followed by organ dysfunction. COVID-19 induced an alteration in coagulation function increase the risk of pulmonary embolism and deep vein thrombosis associated with poor prognosis as well as high mortality. An elevated level D-dimer and other fibrin degrading protein are documented among the patients with COVID-19; especially in severe cases. Differences in coagulopathy among severe and non-severe cases, required prompt attention to adopt a more effective management strategy.
\end{abstract}

Risk assessment and prognostic aspect of coagulopathy in Coronavirus Disease 2019 (COVID19)

Running Title: COVID-19 and blood coagulopathy

\section{Mujibur Rahman, Nadira Naznin Rakhi}

Department of Biotechnology and Genetic Engineering, Bangabandhu Sheikh Mujibur Rahman Science and Technology University, Gopalganj, Bangladesh.

*Correspondence:nadiranrakhi@gmail.com; +8801515241201

Key Word: COVID-19, blood coagulopathy, D-dimer, embolism, thrombosis

To the editor

Coronavirus Disease 2019 (COVID-19) primarily being considered as a respiratory illness has been showing a highly diverse and anomalous array of symptoms since its origin in December 2019. The death toll has already surpassed 327,821 with more than 4.99 million confirmed cases worldwide ${ }^{1}$, while the pathophysiology of COVID-19 is still obscure. At present, concerns are mounting over the increasing reports of blood coagulation accompanied by organ dysfunction among the COVID-19 patients. The disease tends to cause a hyper- and rapid coagulable state allegedly leading to pulmonary embolism and deep vein thrombosis especially among the severe cases of COVID-192. However, predisposition to both venous and arterial thromboembolism causing acute pulmonary embolism (PE), deep-vein thrombosis (DVT), ischemic stroke, myocardial infarction and systemic arterial embolism has also been reported. So, the experts are claiming that COVID-19 causes an eminent change in coagulation function, which is directly associated with disease severity ${ }^{3}$. Even a study showed that $71.4 \%$ of non-survivor COVID-19 patients meet the diagnostic criteria for disseminated intravascular coagulation (DIC) compared to only $0.6 \%$ of the survivors ${ }^{4}$. 
However, that study also reported an elevated level of D-dimer protein among COVID-19 cases, which is produced as the result of fibrinolysis following a thrombotic event and associated with the risk of Acute Respiratory Distress Syndrome (ARDS) ${ }^{5}$. Besides, D-dimer protein was reported significantly higher among the severe cases and the patients requiring intensive care compared to patients with mild symptoms ${ }^{6}$. A meta-analysis on 1015 cases showed a notable difference in D-dimer level along with prothrombin time between severe and mild cases, but not in case of platelet count (PLT) and activated partial thromboplastin time $(\mathrm{aPTT})^{7}$. Most importantly, the level of D-dimer and other fibrin degradation protein of non-survivor cases significantly differ from survivors ${ }^{4}$ and considered as the major cause of mortality. Therefore, the spike in the level of $\mathrm{D}$ - dimer protein provides evidence of abnormal coagulation with the prognostic value, which can be used to evaluate the severity and adverse outcome among patients with community-acquired pneumonia as well as COVID-19 ${ }^{8}$. Moreover, the level of D-dimer has been reported to be dependent on the ethnic groups, which may explain the differential racial susceptibility to COVID-19 severity evident across the world ${ }^{9}$.

However, patients' immobilization during treatment, presence of cardiovascular disease and damage of endothelial cells by viral infection/mechanical procedure has been reported to cause a higher incidence of venous thromboembolism among hospitalized patients ${ }^{10}$, which is even more threatening for COVID-19 susceptible individuals with underlying comorbidities including cardiovascular diseases. Because co-morbidity provokes the COVID-19 severity thus hospitalization, which may lead to thrombotic complications and vice-versa ${ }^{11}$.

So, the differences in coagulopathy, especially D-dimer level among severe and non-severe cases surely urge immediate attention to the current diagnosis and treatment strategy. Besides, both thrombotic complications of COVID-19 and its risk factors need to be addressed to adapt a more effective management strategy.

\section{Acknowledgments}

The corresponding author extends her gratitude to the University Grants Commission (UGC), Bangladesh for funding.

\section{Authors' contributions}

M.R drafted the manuscript. N.N.R developed the hypothesis and critically reviewed the drafted manuscript. All authors read and approved the final manuscript.

\section{Conflict of interest}

The authors declare that the research was conducted in the absence of any commercial or financial relationships that could be construed as a potential conflict of interest.

\section{References}

1. Shanlian L. Index @ Www.Who.Int. Bull World Health Organ . 2018:817-908. http://www.who.int/.

2. Panigada M, Bottino N, Tagliabue P, et al. Hypercoagulability of COVID-19 patients in Intensive Care Unit. A Report of Thromboelastography Findings and other Parameters of Hemostasis. J Thromb Haemost . 2020. doi:10.1111/jth. 14850

3. Han H, Yang L, Liu R, et al. Prominent changes in blood coagulation of patients with SARS-CoV-2 infection. 2020.

4. Tang N, Li D, Wang X, Sun Z. Abnormal coagulation parameters are associated with poor prognosis in patients with novel coronavirus pneumonia. J Thromb Haemost . 2020;18(4):844-847. doi:10.1111/jth.14768

5. Wu C, Chen X, Cai Y, et al. Risk Factors Associated with Acute Respiratory Distress Syndrome and Death in Patients with Coronavirus Disease 2019 Pneumonia in Wuhan, China. JAMA Intern Med . 2020:110. doi:10.1001/jamainternmed.2020.0994

6. Guan WJ, Ni ZY, Hu Y, et al. Clinical Characteristics of Coronavirus Disease 2019 in China. N Engl J Med . 2020. doi:10.1056/NEJMoa2002032 
7. Xiong M, Liang X, Wei Y. Changes in Blood Coagulation in Patients with Severe Coronavirus Disease 2019 (COVID-19): a Meta-Analysis.Br J Haematol . 2020:0-2. doi:10.1111/bjh.16725

8. Snijders D, Schoorl M, Schoorl M, Bartels PC, Werf TS Van Der, Boersma WG. European Journal of Internal Medicine D-dimer levels in assessing severity and clinical outcome in patients with communityacquired pneumonia . A secondary analysis of a randomised clinical trial. Eur J Intern Med . 2012;23(5):436441. doi:10.1016/j.ejim.2011.10.019

9. Fogarty H, Townsend L, Ni Cheallaigh C, et al. COVID-19 Coagulopathy in Caucasian patients. $\mathrm{Br} J$ Haematol . 2020. doi:10.1111/bjh.16749

10. Kahn SR, Lim W, Dunn AS, et al. Prevention of VTE in Nonsurgical Patients Antithrombotic Therapy and Prevention of Thrombosis, 9th ed : American College of Chest Physicians. Chest . 2012;141(2):e195Se226S. doi:10.1378/chest.11-2296

11. Clerkin KJ, Fried JA, Raikhelkar J, et al. Coronavirus Disease 2019 (COVID-19) and Cardiovascular Disease. Circulation . 2020;2019. doi:10.1161/CIRCULATIONAHA.120.046941 